\title{
A nanoflare model of quiet Sun EUV emission
}

\author{
A. Pauluhn ${ }^{1,2}$ and S. K. Solanki ${ }^{1}$ \\ 1 Max-Planck-Institut für Sonnensystemforschung, 37191 Katlenburg-Lindau, Germany \\ e-mail: anuschka.pauluhn@psi.ch \\ 2 now at Paul Scherrer Institut, 5232 Villigen, Switzerland
}

Received 7 March 2006 / Accepted 19 October 2006

ABSTRACT

\begin{abstract}
Nanoflares have been proposed as the main source of heating of the solar corona. However, detecting them directly has so far proved elusive, and extrapolating to them from the properties of larger brightenings gives unreliable estimates of the power-law exponent $\alpha$ characterising their distribution. Here we take the approach of statistically modelling light curves representative of the quiet Sun as seen in EUV radiation. The basic assumption is that all quiet-Sun EUV emission is due to micro- and nanoflares, whose radiative energies display a power-law distribution. Radiance values in the quiet Sun follow a lognormal distribution. This is irrespective of whether the distribution is made over a spatial scan or over a time series. We show that these distributions can be reproduced by our simple model. By simultaneously fitting the radiance distribution function and the power spectrum obtained from the light curves emitted by transition region and coronal lines the power-law distribution of micro- and nanoflare brightenings is constrained. A good statistical match to the measurements is obtained for a steep power-law distribution of nanoflare energies, with power-law exponent $\alpha>2$. This is consistent with the dominant heat input to the corona being provided by nanoflares, i.e., by events with energies around $10^{23} \mathrm{erg}$. In order to reproduce the observed SUMER time series approximately $10^{3}$ to $10^{4}$ nanoflares are needed per second throughout the atmosphere of the quiet Sun (assuming the nanoflares to cover an average area of $10^{13} \mathrm{~m}^{2}$ ).
\end{abstract}

Key words. Sun: flares - Sun: transition region - Sun: corona

\section{Introduction}

The solar emission in the EUV and X-ray wavelength range features transient events on all scales, such as flares and micro- or nanoflares, explosive events, and blinkers, see, e.g., Tandberg-Hanssen \& Emslie (1988) or Solanki (2002) and references therein. Flares, micro- and nanoflares refer to similar phenomena with different magnitudes of energy release, which are usually studied in coronal emission, while explosive events and blinkers are spectral line broadenings and brightenings that are mainly restricted to the transition region. Events in the energy range $10^{30}-10^{33}$ erg $\left(10^{23}-10^{26} \mathrm{~J}\right)$ are usually referred to as "normal" flares. Micro- and nanoflares (Parker 1988) are the brightenings with energy below approximately $10^{27} \mathrm{erg}$, although the limits vary somewhat in the literature. Such transient brightenings are thought to be the signature of dissipation of the energy stored in the magnetic field, e.g., through magnetic reconnection. Thus, they provide a mechanism for heating the solar corona (Parker 1988).

The frequency or rate distribution of the energy of flares $(\mathrm{d} N / \mathrm{d} E)$ has been found to obey a power law for several wavelength regimes. Power laws have also been found for blinkers by Brković et al. (2000) and for explosive events by Winebarger et al. (2002). The distributions found by Winebarger et al. (2002), however, look more consistent with a lognormal function than a power law. Flares and their distributions have been the subject of a large number of studies, e.g., Datlowe et al. (1974); Lin et al. (1984); Krucker \& Benz (1998); Parnell \& Jupp (2000); Aschwanden et al. (2000); Mitra-Kraev \& Benz (2001); Aschwanden \& Parnell (2002); Vekstein \& Jain (2003). Power laws have also been applied for stellar flare energy distributions, see, e.g., Audard et al. (2000); Güdel et al. (2003);
Arzner \& Güdel (2004) and references therein. Lu \& Hamilton (1991) have explained the power-law dependence of the solar flare occurrence rate in a model of self-organized criticality as avalanches of many small reconnection events.

The observed exponents $\alpha$ in the power-law relation $\mathrm{d} N / \mathrm{d} E=E_{0} E^{-\alpha}$ range from 1.5 to 2.9 for solar and for stellar flares, but most studies give values of $\alpha<2$. Exponents within this range are also obtained for blinkers and explosive events. The wide range of the exponent is to a large part due to different selection criteria or assumptions about geometry and structure of the emitting plasma as well as instrumental resolution. The larger the exponent, the more weight is given to smallscale events such as micro- and nanoflares. For an exponent greater than 2 , the energy content is dominated by the smallscale events, and in order to have finite total energy content, a lower cutoff in energy has to be introduced. Since the energy released in flares and flare-like events of sufficient size to be directly detected is not enough to explain the temperature of the corona, an exponent above 2 is necessary if a mechanism producing flaring is to be the main cause of coronal heating. Thus, the distribution of the smallest flares is of great interest in order to have reliable estimates on their participation in coronal heating.

Most previous work has concentrated on identifying individual events in radiance time series. Clearly, it is easy to miss or underestimate the number of the weakest such events, which may lead to a background of almost continuous brightening if they are sufficiently common. Note that in order to judge the amount of energy released through transient events, the X-ray, EUV and UV emission is often decomposed into a (nearly) steady background of emission with superposed transient brightenings. 
Table 1. The SUMER data used in this paper.

\begin{tabular}{ccccc}
\hline \hline Line & Region & Date & Duration/cadence & Remarks \\
\hline O IV $79.0 \mathrm{~nm}$ & TR, $T \approx 10^{5} \mathrm{~K}$ & 8 February 1998 & $7 \mathrm{~h} 20 \mathrm{~min}, 33.5 \mathrm{~s}$ & quiet Sun study, high-telemetry \\
Ne VIII $77.0 \mathrm{~nm}$ & upper TR/lower corona $T \approx 7 \times 10^{5} \mathrm{~K}$ & 8 February 1998 & $7 \mathrm{~h} 20 \mathrm{~min}, 33.5 \mathrm{~s}$ & quiet Sun study, high-telemetry \\
$\mathrm{Si}$ IV $139.3 \mathrm{~nm}$ & TR, $T \approx 10^{5} \mathrm{~K}$ & 19 July 1998 & 3 h 35 min, 15 s & quiet Sun explosive events study \\
\hline
\end{tabular}

In this work we start from the basic assumption that all the emission at EUV wavelengths is produced by transient events (i.e., the observed apparent background is a superposition of many such events). We model in a simple way time series of the radiance for a set of parameters that is consistent with observations. The results are then statistically compared to the observational data.

The main aims of this paper are

I. to identify diagnostics which allow such a statistical comparison to be made. The two diagnostics found and studied are the lognormal probability density function (PDF) of EUV radiances and the wavelet or Fourier power spectrum of radiance time series;

II. to test to what extent the use of these diagnostics can constrain the parameters of the model, in particular the powerlaw exponent $\alpha$ of the distribution of nanoflare amplitudes;

III. to compare with SUMER transition region and coronal data in order to check whether this simple model can reproduce the observations and whether the deduced parameters are realistic.

We begin with a description of the data and their analysis (Sect. 2). Next we give an outline of the model used for the flare simulation and establish a heuristic reasoning for a lognormal distribution of the radiances under the assumption that they are entirely due to transient events (Sect. 3). The results of parameter studies with our model are presented in Sect. 4. In Sect. 5 we compare the simulations with SUMER measurements, and we outline our conclusions in Sect. 6.

\section{The SUMER data: reduction and analysis}

\subsection{Data and reduction}

SUMER is a stigmatic normal incidence telescope and spectrometer, operating in the wavelength range from 46.5 to $161.0 \mathrm{~nm}$, depending on the spectral order and the choice of detector. For a general description of the SUMER instrument and its data we refer to Wilhelm et al. (1995). The SUMER slit with angular dimensions of $1^{\prime \prime} \times 300^{\prime \prime}$ used for the data analyzed here is imaged by the spectrograph on to the detectors with a resolution of about $1^{\prime \prime}$ per pixel in the spatial direction and $4.4 \mathrm{pm}$ per spectral pixel in first order and $2.2 \mathrm{pm}$ per spectral pixel in second order.

The O IV line at $79.0 \mathrm{~nm}$ and the Ne VIII line at $77.0 \mathrm{~nm}$, which are used here, are measured in first order. We also analyzed and modelled the Si Iv line at $139.3 \mathrm{~nm}$, but the results were very similar to those pertaining to the O IV line and are not described in detail in the following. On 8 February 1998, SUMER performed a long-duration observation of quiet areas near disk centre in the O IV and Ne VIII lines. The measurements, which have been described by Wilhelm \& Kalkofen (2003), were taken over $7 \mathrm{~h}$ and $20 \mathrm{~min}$ with a cadence of $33.5 \mathrm{~s}$ and a particularly accurate compensation of the solar rotation. The Si IV measurements, recorded 19 July 1998, belong to a quiet Sun explosive events study. Recordings were made over $3 \mathrm{~h}$ and $35 \mathrm{~min}$ with a cadence of $15 \mathrm{~s}$. (See Table 1 for a summary of the measurements.)

The data were corrected for the flatfield, the geometric distortion, and for detector electronics effects such as dead-time and local-gain depression.

After the instrumental corrections and the radiometric calibration, the solar radiances were determined by integration over the line profiles, which were derived by least-squares fits of single Gaussian functions and a linear background. The spectral background (continuum) was subtracted prior to integration.

The data used by Pauluhn et al. (2000) were mainly image scans and thus gave the distribution of the radiances in the scanned area during the time span of the image recording. They may be considered to give a snapshot of the distribution of radiances. Here, we investigate time series, i.e., we focus on the distribution of radiances in a certain area caused by temporal variations.

\subsection{Data analysis: lognormal distribution}

Before doing any modelling we investigate whether time series of quiet Sun radiances are approximated by a lognormal distribution. After finding that this is the case, we determine the parameters of the distribution, which is characterised by the following probability density function $\rho$ for an independent parameter $x$ (e.g., the radiance)

$\rho(x)=\frac{N_{0}}{\sigma x \sqrt{2 \pi}} \exp \left(-\frac{(\log (x)-\mu)^{2}}{2 \sigma^{2}}\right)$,

with $\mu=\langle\log (x)\rangle, \sigma=\sqrt{\operatorname{Var}(\log (x))}$ and $N_{0}$ a normalization factor. Var means the variance. $\sigma$ is called the shape parameter because it determines the shape of the distribution: small $\sigma$ makes the distribution more Gaussian-like, large $\sigma$ makes the distribution more skewed. $\mu$ is called the scale parameter and stretches the distribution function.

An example histogram of a SUMER time series of the transition region ( $\mathrm{Si}$ IV line at $139.3 \mathrm{~nm}$ ) is shown in Fig. 1, along with a lognormal fit. The bin size is $5 \%$ of the maximum radiance value. For practically all spatial pixels the distributions of the $\mathrm{O}$ IV, the $\mathrm{Si}$ IV, and the $\mathrm{Ne}$ VIII radiances are well described by lognormal functions. This does not automatically follow from the result of Pauluhn et al. (2000), since they considered the distribution of radiance from different spatial locations, which represented a mix of network and cell interior locations. Also, the lognormal distributions from network and cell deduced from the time series differ in their $\mu$ values, but not significantly in their $\sigma$ values (see Sect. 5).

At coronal temperatures the shapes of the probability density functions are narrower than for the transition region and more symmetric (cf., Pauluhn et al. 2000), and the time series shows peaks that are less well marked, which will be shown in Sect. 5. Thus the difference between the distributions of radiances reflecting different temperatures is equally shown by data sampling a part of the solar surface or a single point over a time 


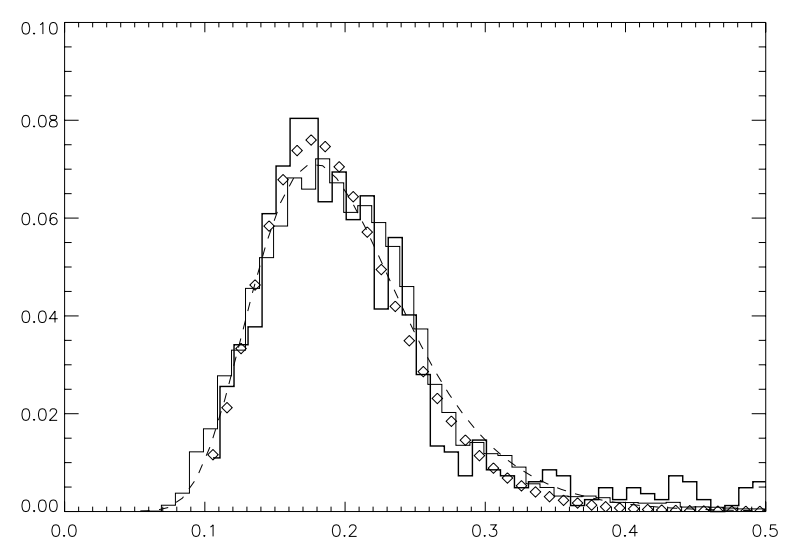

Fig. 1. Example of a histogram from a SUMER transition region time series (quiet area, $\mathrm{Si}$ Iv line at $139.3 \mathrm{~nm}$ ). The radiance data and the corresponding lognormal fit are given by the thick-line histogram and diamonds, and a simulation and its fit are indicated by the thin-line histogram and a dashed curve.

interval. The $\mu$ and $\sigma$ values found from fits to the observations are therefore useful constraints to any model of quiet-Sun radiances.

\section{A simple model}

\subsection{Description}

Using a simple model of transient brightenings, we produce synthetic time series of EUV radiances. We presume that flaring is an intrinsically stochastic process, and our radiance variable is a time-dependent random variable. One simulation thus delivers a possible realization of this process.

Our model consists of a time series of random kicks (acting as "flares"), applied to an initial radiance. Each kick is followed by the exponential decay of the radiance. The final radiance is given by the sum of the radiances of all the overlapping transient brightenings. The numerical value of the initial radiance is not important, since it is damped just like every brightening and the model reaches a statistical "steady state" after a certain relaxation time. We have 5 free parameters: the maximum and minimum allowed flare amplitude, $y_{\max }$ and $y_{\min }$, the power-law exponent $\alpha$, the e-folding or damping time of the flare $\tau_{\mathrm{d}}$, and the frequency of the excitation, i.e., the flaring rate or flaring probability $p_{\mathrm{f}}$. This set is complete if we assume that the shape of the nanoflare is given by a single kick with a sharp rise and a successive exponential decrease. These parameters are sufficient to quantitatively reproduce the radiance PDFs and power spectra. In order to smooth the steep increase a rise time $\tau_{\mathrm{r}}$ can be introduced (which, if included, is chosen to be a fixed fraction of the damping time and hence does not add an additional free parameter). The introduction of a rise time improves the qualitative agreement between synthetic and observed time series.

For the energy content of a flaring event of amplitude $y_{0}$ and damping time $\tau_{\mathrm{d}}$ the following proportionality holds

$E \sim \int_{t_{0}}^{\infty} y_{0} \mathrm{e}^{-\frac{\left(t-t_{0}\right)}{\tau_{\mathrm{d}}}} \mathrm{d} t=y_{0} \tau_{\mathrm{d}}$.

Thus, the energy content of a flare with amplitude $y_{0}$, damping time $\tau_{\mathrm{d}}$ and rise time $\tau_{\mathrm{r}}$ can be estimated as

$E=q y_{0} \pi A\left(\tau_{\mathrm{d}}+\tau_{\mathrm{r}}\right)$ where $A$ is the solar surface area, if we assume equal radiation in all directions (Lambertian surface), and $q$ is a fraction of the total energy radiated in the observed spectral line. In general $q$ is a small number, determining whose exact value is beyond the scope of the present paper.

\subsection{The simulation}

The model produces realizations of possible radiance time series, i.e., we get the radiance as a time-dependent stochastic variable (stochastic process), and it describes an example of a process which is close to being Markovian. In a Markov process, the stochastic variable at one timestep $t_{n+1}$ is only dependent on the directly preceding timestep $t_{n}$. It has "nearly no memory" of the history, and the probability to find a variable at position $x_{n+1}$ at time $t_{n+1}$ is calculated by an initial probability and the two-time transition probability (which is the conditional probability that the variable is in state $x_{n+1}$ at $t_{n+1}$ under the condition that it has been in state $x_{n}$ at $t_{n}$ ).

The simulation involves the following steps:

1. Generate a distribution of flare strengths, i.e. (positive) values of flare amplitudes $f_{n}$.

2. Start from an initial radiance value $r_{0}>0$ (the "first kick", taken from the flare distribution, $r_{0}=f_{0}$ ).

3. At random time $t_{i}$ another radiance $r_{i}$ is generated by adding a flare kick $f_{i}$,

$r_{i}=r_{i-1}+f_{i}$.

The random amplitude $f_{i}$ is required to be consistent with the chosen distribution function.

4. At successive time steps $t_{j}, j>i$, the radiance values are

$r_{j}=r_{i} \cdot \exp \left(-\frac{t_{j}-t_{i}}{\tau_{\mathrm{d}}}\right)$

with $\tau_{\mathrm{d}}$ the damping time, corresponding to a difference equation of

$r_{j}-r_{j-1}=r_{j-1}\left(\exp \left(-\frac{t_{j}-t_{j-1}}{\tau_{\mathrm{d}}}\right)-1\right)$,

multiplicatively generated from the preceding values. Here we assume for simplicity that all brightening events have the same damping time.

The probability of a transient brightening occurring (per time step), $p_{\mathrm{f}}$ with $0<p_{\mathrm{f}}<1$, is simulated by drawing equally distributed random numbers between 0 and 1 , and a flare event is started at $t_{i}$ if the random number falls within a certain fraction of the interval $(0,1)$. For example, a flaring probability of $30 \%$ or 0.3 is realized by applying the kick if the random number is smaller than 0.3 . The flare process is thus a Poisson process, which is equivalent to the fact that the waiting times, i.e., the time intervals between two flares $\Delta T_{i}$, are exponentially distributed with parameter $p_{\mathrm{f}}$, and the mean waiting time $\langle\Delta T\rangle$ is given by $1 / p_{\mathrm{f}}$. Additionally to the excitation, we include a damping process with damping time $\tau_{\mathrm{d}}$, which is inverse to the strength of the damping process. In Fig. 2 an example sequence of flares with very large mean waiting times or low frequency $p_{\mathrm{f}}$ is shown. For higher frequencies, the radiances due to individual "kicks" or flare-like events overlap much more.

A heuristic explanation for the distribution of the radiances is given in the following. We assume that we can express the kick as $f_{i}=c \cdot r_{i-1}$, with $c$ a positive value, i.e., the new radiance is

$r_{i}=r_{i-1}+c \cdot r_{i-1}=\hat{c} \cdot r_{i-1}$, 


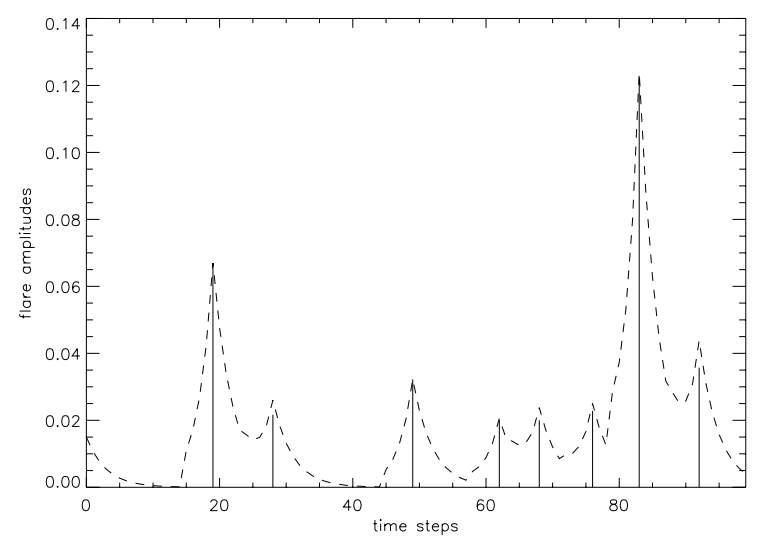

Fig. 2. An example sequence of simulated flares. Here, the flaring frequency has been chosen to be very small, $p_{\mathrm{f}}=0.08$, such that the average waiting time between two flares is 12.5 time steps.

a multiple of the predecessor radiance. We stress, however, that we prescribe the distribution of the additive components $f_{i}$ (given, e.g., by observation as a power law) and not that of the multiplicative component $c$.

Thus, the radiance value at the $n$th step is

$r_{n}=r_{0} \cdot c_{1} \cdot c_{2} \cdots c_{n}$

with the $c_{i}$ being random factors if a new kick happens at this time. Otherwise $c_{i}$ represents the damping as described by the exponential in Eq. (5).

This assumption of a multiplicative process is plausible in the light of the radiance being ultimately generated by emerging and decaying magnetic field. This is for example implied by the excellent correlation between high-resolution magnetograms and images in EUV and X-ray wavelengths, although the underlying processes are still far from being understood.

Taking the logarithm of Eq. (8) yields

$\log \left(r_{n}\right)=\log \left(r_{0}\right)+\log \left(c_{1}\right)+\log \left(c_{2}\right)+\cdots+\log \left(c_{n}\right)$.

The Central Limit Theorem then states that as $n$ goes to infinity, the distribution of the sum converges to a normal distribution. For the Central Limit Theorem to hold, the $c_{i}$ have to be independent random variables, which is not precisely fulfilled here, because the flaring process is coupled via the damping time scale and thus has a (short) memory. We can, however, assume that the damping time is short relative to the other time scales of the system, e.g., the duration of the measurements, which would link the flare process to a coloured noise process instead of a white noise process (see, e.g., Honerkamp 1990).

Thus, the distribution of the sum in Eq. (9) is approximately normal, so that the distribution of the $r_{n}$ is approximately lognormal.

On the time scales under study (several hours), we can assume stationarity of our distribution, which means that (after an initial relaxation time) a steady state is reached. This does not hold for the solar radiance when the structure of the region changes significantly, e.g., due to emergence of new flux and thus a change in activity. Our study is thus limited to truly quiet regions.

The two characterizing parameters of the stationary solution Eq. (1), $\mu$ and $\sigma$, have been determined empirically by parameter scans for Gaussian and for power-law stochastic flare input.



Fig. 3. Histograms of the simulations with $\tau_{\mathrm{d}}=10,50,100$, and 200 time steps for power-law flare distributions with fixed flaring probability of 0.2 .

\section{Parameter studies}

In this section we describe the effects of varying the free parameters of the simulation on the parameters of the radiance distribution. The simulated time series consisted of $n=5 \times 10^{5}$ time steps each.

First we need to demonstrate that the distribution function of the radiance has a lognormal shape. We ran the model for flare amplitudes which are distributed according to a power law, with the exponent $\alpha=2.1$ and the amplitude ranging between 0.02 and $3.0 \mathrm{~W} \mathrm{~m}^{-2} \mathrm{sr}^{-1}$. Figure 3 shows examples of simulated distributions with damping times $\tau_{\mathrm{d}}=10,50,100$ and 200 time steps, and a flaring frequency of $p_{\mathrm{f}}=0.2$ (in inverse time steps). Clearly, the resulting distributions are highly skewed towards small radiances for a short damping time. In the limit of infinitely short damping time the most common value of the radiance is zero. As the damping time increases, so do the average value and the width of the radiance distribution, which also becomes increasingly symmetric. The same behaviour is found as the flaring frequency increases (not plotted). Note that for increasing $\tau_{\mathrm{d}}$, as the overlap between individual brightenings increases, a background of nearly constant brightness is built up. This becomes visible in Fig. 3 through the increasing amount of points at small radiances at which the PDF is zero.

In order to check if a lognormal results only for a power law we also considered, as a test case, a Gaussian distribution of flares, which results in a far more symmetric distribution of radiances than when the distribution of flare amplitudes is a power law. Nevertheless, it is also well approximated by a lognormal distribution. In the following we consider only the power-law distribution of flare amplitudes, since it is supported by observation.

\subsection{Variation of the damping time and flaring frequency}

The shape of the equilibrium distribution is generally determined by the ratio of damping to excitation. Only when the two forces balance each other, can a stationary state be reached. To confirm this for our simulations, we vary the quotient of damping strength and excitation frequency $\frac{1}{\tau_{\mathrm{d}} p_{\mathrm{f}}}$ in two different ways: first, we vary $\tau_{\mathrm{d}}$ and allow $p_{\mathrm{f}}$ to remain constant. With increasing damping time, the shape parameter $\sigma$ decreases, which means 


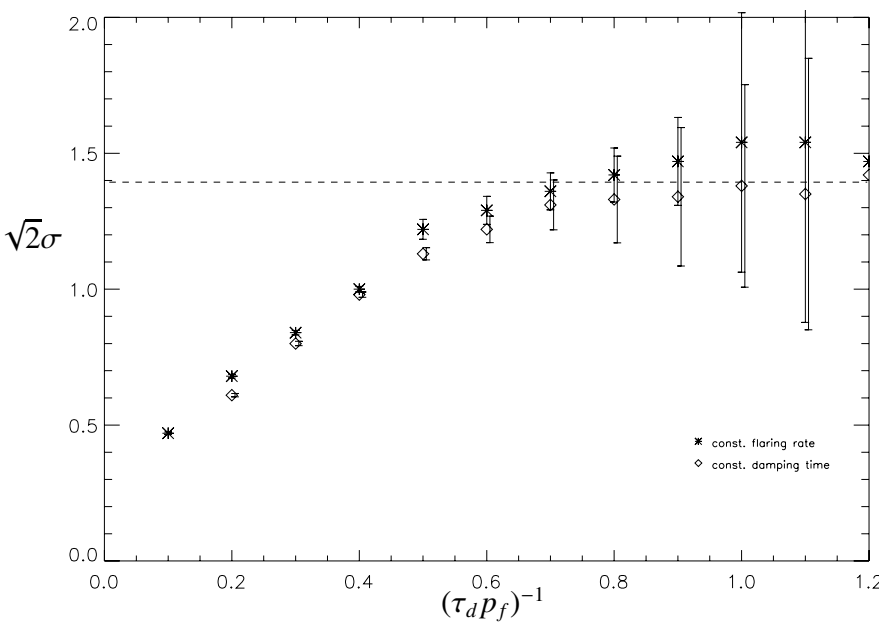

Fig. 4. Variation of the lognormal shape parameter with the ratio of damping to flaring rate. The stars give the results for constant flaring rate $p_{\mathrm{f}}$ and varying damping time $\tau_{\mathrm{d}}$, and the diamonds give the results for constant $\tau_{\mathrm{d}}$ and varying $p_{\mathrm{f}}$. The horizontal dashed line represents the observed value as deduced from SUMER data.

that the skewness of the lognormal decreases. The distribution becomes more symmetric when the damping is weaker. Then we do the opposite and vary $p_{\mathrm{f}}$ while leaving $\tau_{\mathrm{d}}$ unchanged. The results (for the parameters $\alpha=2.5, y_{\min }=0.016, y_{\max }=0.8$ ) are shown in Fig. 4. Both curves agree with each other within the uncertainties of the fits. From Fig. 4 it follows

$$
\sqrt{2} \sigma=\frac{K_{\alpha}}{\sqrt{\tau_{\mathrm{d}} p_{\mathrm{f}}}}+\text { const. }
$$

The proportionality factor $K_{\alpha}$ varies between values of 1 and 2 . As either $\tau_{\mathrm{d}}$ or $p_{\mathrm{f}}$ increase the radiance enhancements due to consecutive kicks increasingly overlap, leading to an increasingly symmetric distribution. For very low $\tau_{\mathrm{d}}$ and $p_{\mathrm{f}}$ low radiances dominate the distribution, while the few high peaks make it very asymmetric (see Fig. 3). Also empirically, we find the following dependence for $\mu$ :

$\mu=\log \left(\tau_{\mathrm{d}} m_{\mathrm{f}} p_{\mathrm{f}}\right)+p_{\mathrm{f}} \exp \left(p_{\mathrm{f}}\right)+$ const.

where $m_{\mathrm{f}}$ is the mean value of the flare amplitudes.

Where the damping or excitation exceed certain limiting values, no stable solution exists that can be described by a lognormal distribution. If the excitation is too strong (for this set of parameters at $\frac{1}{\tau_{\mathrm{d}} p_{\mathrm{f}}} \leq 0.1$ ), the realizations grow unboundedly, and at the other end of the range, if the product $\frac{1}{\tau_{\mathrm{d}} p_{\mathrm{f}}}$ exceeds a certain value (here 1.2), the shape of the radiance distribution changes from a two-sided function to a function with a maximum value at or very near zero and a rightwards extending tail.

\subsection{Dependence on the minimum and maximum flare amplitude}

For constant flaring frequency and damping time the energy input to the system is determined by the flare distribution, i.e., the minimum and maximum flare amplitude and the exponent of the power law. For fixed upper and lower boundaries, the energy input decreases with increasing exponent. Thus, in order to reproduce a certain (measured) radiance time series and its mean value, the boundary $y_{\min }$ and/or $y_{\max }$ has to be varied

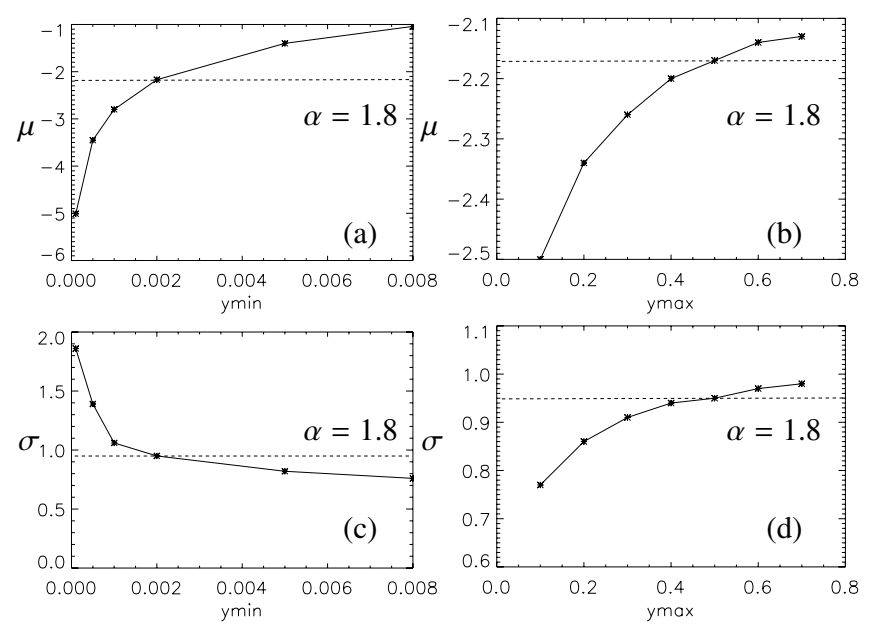

Fig. 5. Variation of the lognormal scale and shape parameters with the lower a), c) and upper b), d) cutoffs of the flare amplitude distribution. The values employed for the other parameters are $\alpha=1.8$, $\tau_{\mathrm{d}}=8.01, p_{\mathrm{f}}=0.60,\left(y_{\min }\right.$ variable, $\left.y_{\max }=0.5\right)$; and $\left(y_{\max }\right.$ variable, $\left.y_{\min }=0.002\right)$. The horizontal dashed lines represent observed values (averages over O Iv quiet network area).

simultaneously with the exponent such as to keep the energy input constant. The mean of the flare radiance input is given by

$$
\begin{gathered}
m_{\mathrm{f}}=E_{0} \int_{y_{\min }}^{y_{\max }} E E^{-\alpha} \mathrm{d} E=E_{0} \int_{y_{\min }}^{y_{\max }} E^{1-\alpha} \mathrm{d} E \\
\text { with } E_{0}=\left(\int_{y_{\min }}^{y_{\max }} E^{-\alpha} \mathrm{d} E\right)^{-1} \\
=\frac{(1-\alpha)}{(2-\alpha)} \frac{\left(y_{\max }^{2-\alpha}-y_{\min }^{2-\alpha}\right)}{\left(y_{\max }^{1-\alpha}-y_{\min }^{1-\alpha}\right)}
\end{gathered}
$$

Figure 5 shows the dependence of the lognormal parameters on the minimum and the maximum flare amplitude for $\alpha=1.8$. We find that the distribution of the radiances depends more strongly on the lower energy limit of the nanoflares than on the upper limit also for other $\alpha$ values, including $\alpha>2$. The mean of the resulting radiance values varies as the mean of a lognormal function

mean $_{\operatorname{logn}}=\mathrm{e}^{\mu+\frac{\sigma^{2}}{2}}$

\subsection{Dependence on the power-law exponent}

Figure 6 shows the probability densities and cumulative distribution functions (CDFs) of the simulated time series for different values of $\alpha$. Also plotted are the same quantities from the time series of the transition region O IV $79.0 \mathrm{~nm}$ line recorded by SUMER in the quiet network. The histograms have been computed over the ensemble of all network time series in the observed quiet-Sun area (60 pixels). They have been calculated with a binsize of $0.01 \mathrm{~W} \mathrm{~m}^{-2} \mathrm{sr}^{-1}$. The exponent $\alpha=2.5$ yielded the best match to the selected SUMER data. The quality of the fit was determined from a comparison of the density functions fitted to the histograms and the corresponding cumulative distribution functions. However, this diagnostic does not allow values of $\alpha \geq 2.5$ to be distinguished from each other unless the quality of the data is high and the statistics are good. In Fig. 7 we show 

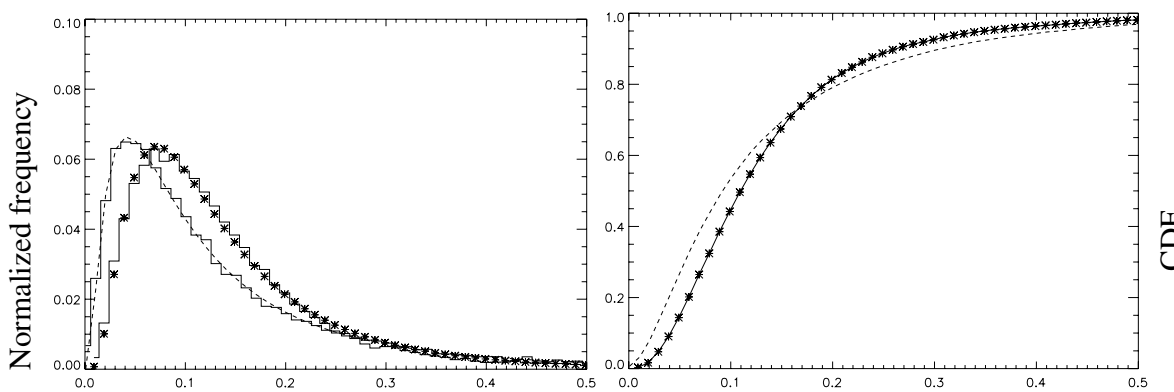

它

$\alpha=1.8$
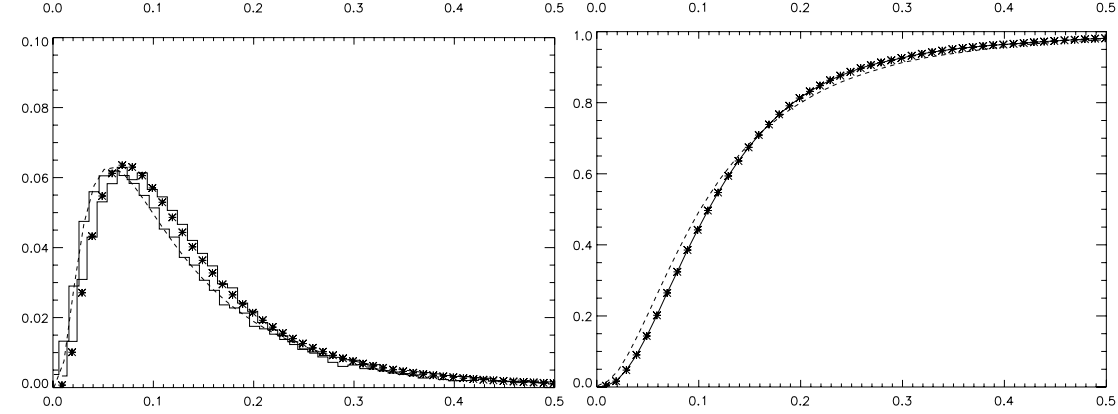

$\alpha=2.1$
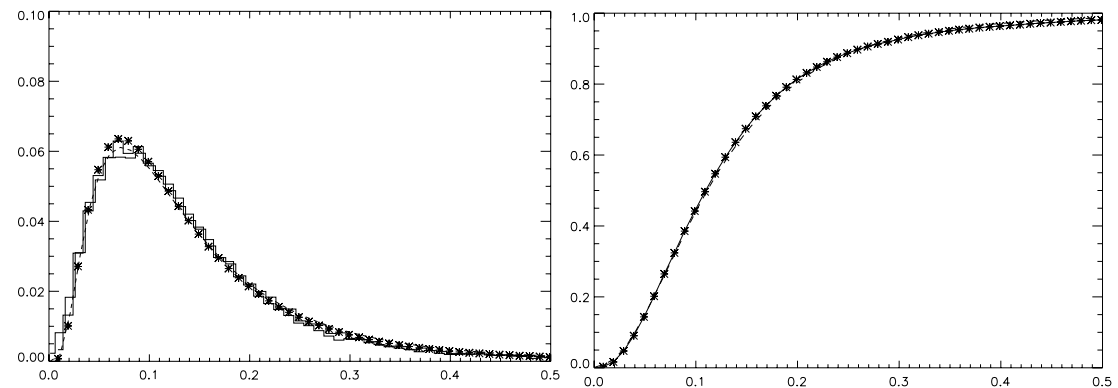

$\alpha=2.5$
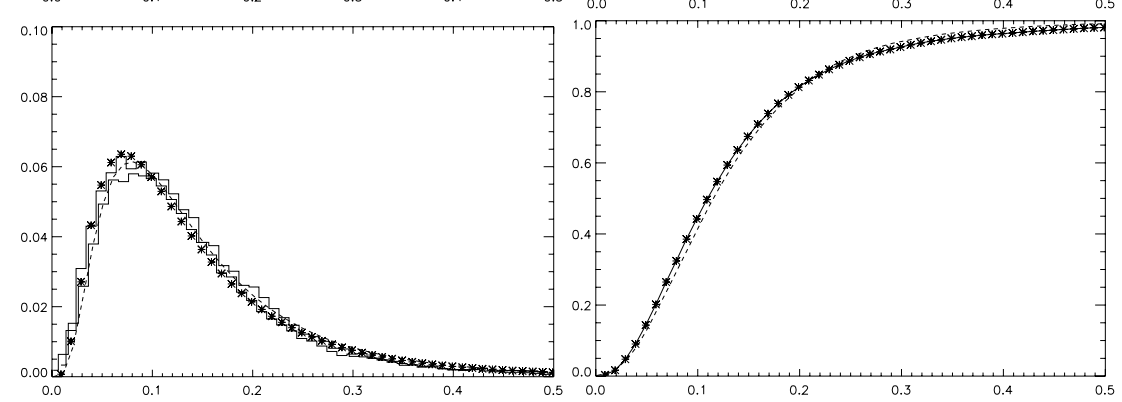

$\alpha=2.8$
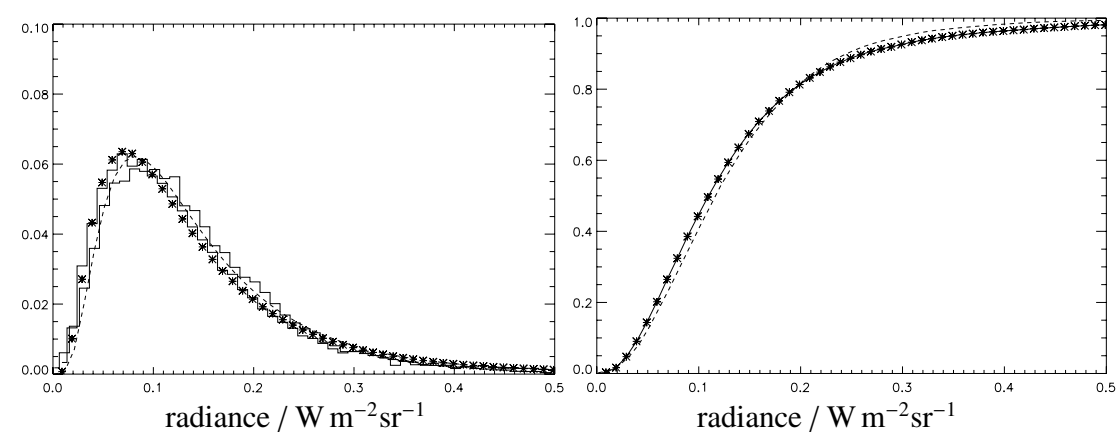

$\alpha=3.0$

Fig. 6. Variation of the radiance distribution (the radiance probability density function, shown on the left, and its cumulative distribution function, shown on the right) with the exponent $\alpha$ of the flare distribution. The following parameters have been kept constant: $p_{\mathrm{f}}=0.43, \tau_{\mathrm{d}}+\tau_{\mathrm{r}}=5.72$ time steps, and $y_{\max }=0.8$. The simulated histogram is represented by the thin solid line. The dashed line indicates the lognormal-based fit to this distribution. Also shown are a SUMER O Iv histogram representative of the network and the corresponding lognormal fit (stars).

the lognormal parameters $\mu$ and $\sigma$ as a function of $\alpha$ for constant flare frequency $p_{\mathrm{f}}=0.43\left(1.3 \times 10^{-2} \mathrm{~Hz}\right)$, damping time $\tau_{\mathrm{d}}+\tau_{\mathrm{r}}=5.72$ time steps $(191.7 \mathrm{~s})$ and constant upper amplitude value of $y_{\max }=0.8$, the value of $\alpha$ has been varied while also varying the $y_{\min }$ value $\left(y_{\min }=0.002,0.006,0.011,0.016\right.$,
$0.019,0.021$, respectively), such as to keep the energy input constant (as well as the number of flares). Clearly, the distribution of radiances becomes more symmetric when $\alpha$ increases (when the amplitude range is selected in order to keep the mean value constant). It is also evident from Fig. 6 that the sensitivity of 

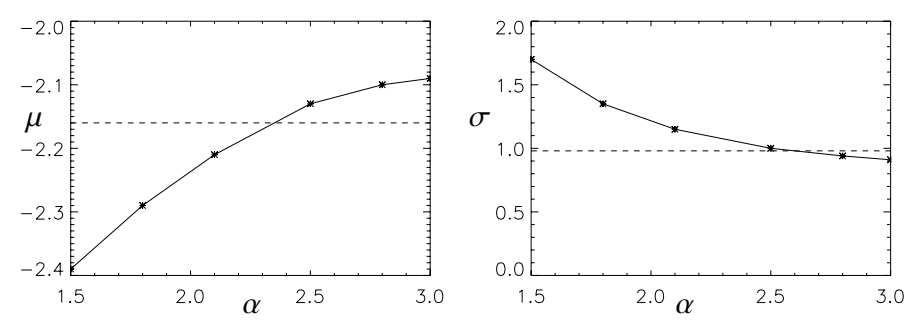

Fig. 7. Variation of the lognormal parameters with the exponent $\alpha$ of the flaring input distribution. The total energy input has been held constant by varying the lower boundary of the flare amplitudes accordingly. The dashed lines show the corresponding lognormal-fit values to the averaged SUMER O Iv network data.

Table 2. Values of $\alpha, p_{\mathrm{f}}, \frac{1}{\tau_{\mathrm{d}} p_{\mathrm{f}}}, y_{\min }$ and $y_{\max }$, as well as the $\mu, \sigma$, and mean of the distributions produced by them. The values of the SUMER time series used for comparison (O IV network) were $\mu_{\mathrm{SUM}}=$ $-2.16, \sigma_{\mathrm{SUM}}=0.98$, and mean SUM $=0.15 . \Delta C D F$ gives the summed difference of the cumulative distribution functions.

\begin{tabular}{cccccc}
\hline \hline$\alpha$ & 1.8 & 2.1 & 2.5 & 2.8 & 3.0 \\
\hline$\tau_{\mathrm{d}}+\tau_{\mathrm{r}}$ & 8.01 & 6.87 & 5.72 & 5.66 & 5.55 \\
$p_{\mathrm{f}}$ & 0.60 & 0.52 & 0.43 & 0.43 & 0.42 \\
$\frac{1}{\tau_{\mathrm{d}} p_{\mathrm{f}}}$ & 0.21 & 0.28 & 0.41 & 0.41 & 0.43 \\
$y_{\min }$ & 0.002 & 0.006 & 0.016 & 0.019 & 0.021 \\
$y_{\max }$ & 0.5 & 0.6 & 0.8 & 0.8 & 0.8 \\
\hline$\mu$ & -2.17 & -2.21 & -2.12 & -2.12 & -2.16 \\
$\sigma$ & 0.95 & 0.97 & 1.0 & 0.97 & 0.95 \\
mean & 0.15 & 0.14 & 0.15 & 0.15 & 0.14 \\
$\Delta C D F$ & 0.007 & 0.008 & 0.004 & 0.007 & 0.013 \\
\hline
\end{tabular}

the radiance distribution to variations of $\alpha$ is highest for small $\alpha$ values. Figure 7 confirms the result obtained from Fig. 6 that $\alpha=2.5$ agrees best with the SUMER data.

\subsection{The power spectrum as an additional diagnostic}

Not surprisingly, just the probability density function alone does not lead to a unique solution, since the PDF contains no information on time scales. This is illustrated in Figs. 8-10. In Fig. 8 we show the observed and simulated PDFs. The observed PDF of this network time series is very similar to that of the mean which is shown in Fig. 6. Obviously, the chosen parameters of this particular simulation $\left(\alpha=2.24, \tau_{\mathrm{d}}+\tau_{\mathrm{r}}=19.1\right.$ time steps, $p_{\mathrm{f}}=0.16$, $\left.y_{\min }=0.014, y_{\max }=0.76\right)$ give an excellent fit to the observed PDF. A comparison with an actual time series (Fig. 9) shows, however, that the individual microflares last much longer in the simulations than in the data. Therefore, the probability distribution cannot constrain the dynamics of the time series. In terms of the free parameters of the simulation, this implies that multiple combinations of free parameters can produce the same probability distribution, i.e., the same $\mu$ and $\sigma$ values. To demonstrate this we have varied $\alpha$ and $\frac{1}{\tau_{\mathrm{d}} p_{\mathrm{f}}}$ together, such that for smaller $\alpha$ we have chosen a higher $\frac{1}{\tau_{\mathrm{d}} p_{\mathrm{f}}}$ in order to keep the $\sigma$ near the observed value. The observed values of the $\mu$ and $\sigma$ parameters are generated using all the sets of simulation parameters given in Table 2.

The dynamics of the time series corresponding to the parameters given in Table 2, however, are in general not identical. In particular, $\tau_{\mathrm{d}}$ and $p_{\mathrm{f}}$ influence the duration of the individual events and the interval between them. In order to constrain the time dependence, a diagnostic sensitive to time scales

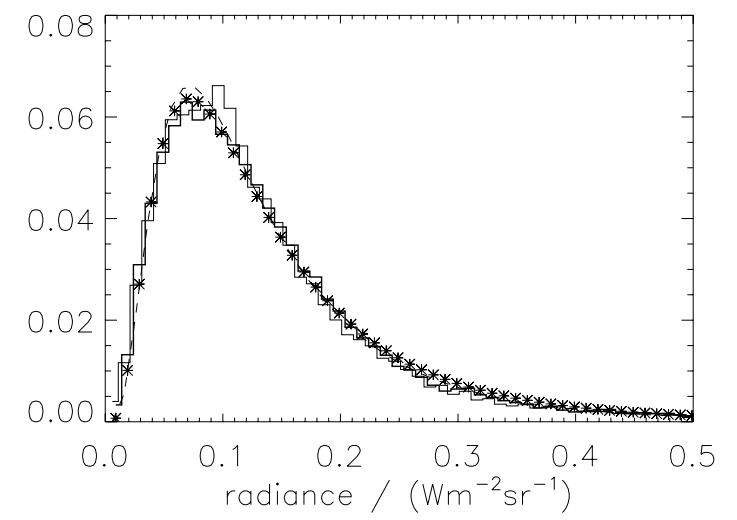

Fig. 8. The histograms corresponding to the time series of Fig. 9. The simulated histogram is represented by the thin solid line. The dashed line indicates the lognormal-based fit to this distribution. The SUMER histogram is given by the thick line and its fit is indicated by the stars.
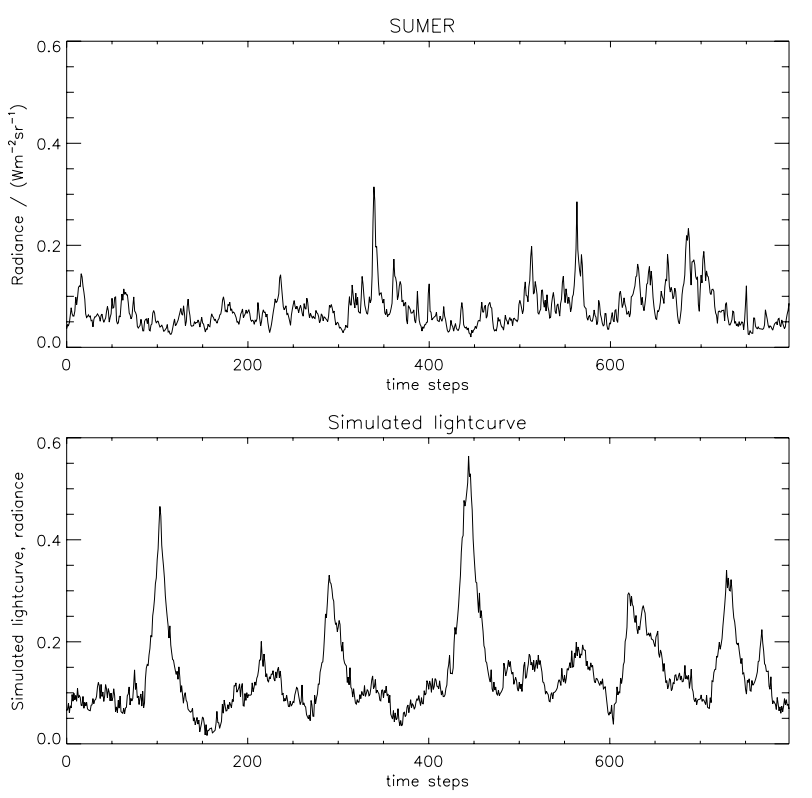

Fig. 9. Example of a simulation featuring a similar lognormal distribu-

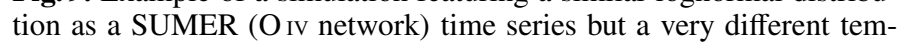
poral variation, so that the time series look rather different. Parameters of the simulation are $\alpha=2.24, \tau_{\mathrm{d}}+\tau_{\mathrm{r}}=19.1$ time steps, $p_{\mathrm{f}}=0.16$, $y_{\min }=0.014, y_{\max }=0.76$. The corresponding histograms are shown in Fig. 8 .

of radiance variation is required. The (Fourier or wavelet) power spectrum is such a diagnostic. The power spectral density was computed here using (Morlet-)wavelets (see, e.g., Torrence \& Compo 1998). This choice is driven by the smaller amount of fluctuations in this quantity than in Fourier power spectra, since wavelets introduce a smoothing in the spectral domain. In Fig. 10 we note that the main peak of the global wavelet power spectrum of the observed time series lies at nearly one half of the frequency of that of the model. Thus, this diagnostic nicely complements the radiance distribution. As we show in Sect. 5 the combination of distribution function and power spectrum leads to much stronger constraints on the free parameters of the model. We cannot rule out, however, that the distinct peaks seen in the power spectra are partly due to the restricted length of the time series and the thereby limited statistics. Moreover, some properties of the flaring process are best seen by directly comparing 
Table 3. Best-fit parameters for the SUMER measurements of 8 February 1998.

\begin{tabular}{ccccccccc|ccc|c}
\hline \hline & $\alpha$ & $\tau_{\mathrm{d}}+\tau_{\mathrm{r}}$ & $p_{\mathrm{f}}$ & $y_{\min }$ & $y_{\max }$ & $\mu$ & $\sigma$ & mean & $\mu_{\text {SUM }}$ & $\sigma_{\text {SUM }}$ & mean $_{\text {SUM }}$ & $\Delta C D F$ \\
\hline dark px. 79.0 nm & 2.5 & $5.721(191.68 \mathrm{~s})$ & 0.43 & 0.004 & 0.80 & -3.54 & 1.08 & 0.042 & -3.58 & 1.09 & 0.039 & 0.0045 \\
bright px. 79.0 nm & 2.5 & $5.721(191.68 \mathrm{~s})$ & 0.43 & 0.016 & 0.80 & -2.12 & 1.0 & 0.15 & -2.16 & 0.98 & 0.15 & 0.0043 \\
dark px. 77.0 nm & 2.5 & $44.40(1487.5 \mathrm{~s})$ & 0.11 & 0.003 & 0.01 & -3.67 & 0.47 & 0.027 & -3.67 & 0.45 & 0.028 & 0.0005 \\
bright px. 77.0 nm & 2.5 & $18.28(612.5 \mathrm{~s})$ & 0.43 & 0.003 & 0.01 & -2.86 & 0.39 & 0.06 & -2.84 & 0.39 & 0.06 & 0.0022 \\
\hline
\end{tabular}

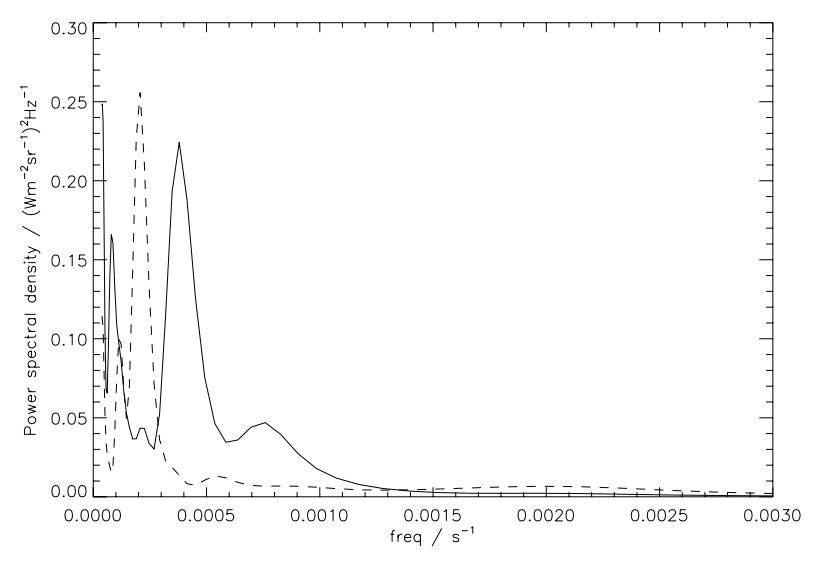

Fig. 10. The global wavelet power spectra of the modelled (solid line) and the observed (dashed) time series corresponding to Figs. 9 and 8.

with an observed time series, although this comparison remains qualitative.

\section{Application of the model to SUMER transition region and coronal data}

SUMER data of the EUV lines O IV, Si IV and Ne VIII were used to constrain the free parameters of the model. These lines represent temperatures of the lower (cooler) transition region ( $\mathrm{O}$ IV, $\mathrm{Si}$ IV) and the upper (hotter) transition region or lower corona (Ne VIII). We restricted ourselves to areas where no significant change of the activity was discernible, i.e., truly quiet regions with no detectable trend in the average radiance during the measurements. The parameter values obtained from these data are listed in Table 3. This table summarizes the optimum parameters for our model to represent the averages over the cell and network time series as suggested by comparison with the SUMER data. The summed difference of the cumulative distribution functions (compare Fig. 6) is given in the last column.

The model parameters were varied until a good (statistical) match was obtained to the SUMER time series, its radiance distribution and its global wavelet power spectrum. We used a statistical power-law distributed flaring process and introduced a possible rise time of a flare $\tau_{\mathrm{r}}$, in order to better match the observed time series. The flare brightness was chosen to both, rise and fall exponentially. In all cases, $\tau_{\mathrm{r}}$ was chosen to be $75 \%$ of the damping time $\tau_{\mathrm{d}}$, so that the flaring time scale or "effective damping time" was $\tau_{\mathrm{r}}+\tau_{\mathrm{d}}$. Note that the need for a rise time $\tau_{\mathrm{r}}$ comes neither from the radiance distribution functions nor from the power spectrum, but rather is indicated by the direct comparison between the time series (such as those shown in Figs. 12 to 17). Each simulation consisted of $n=24000$ time steps. For the radiance histograms, a bin size of approximately $5 \%$ of the respective maximum value has been used.

We found that adding Poisson photon noise $(\sqrt{\text { counts }})$ to the model realizations gives slightly better matches when comparing
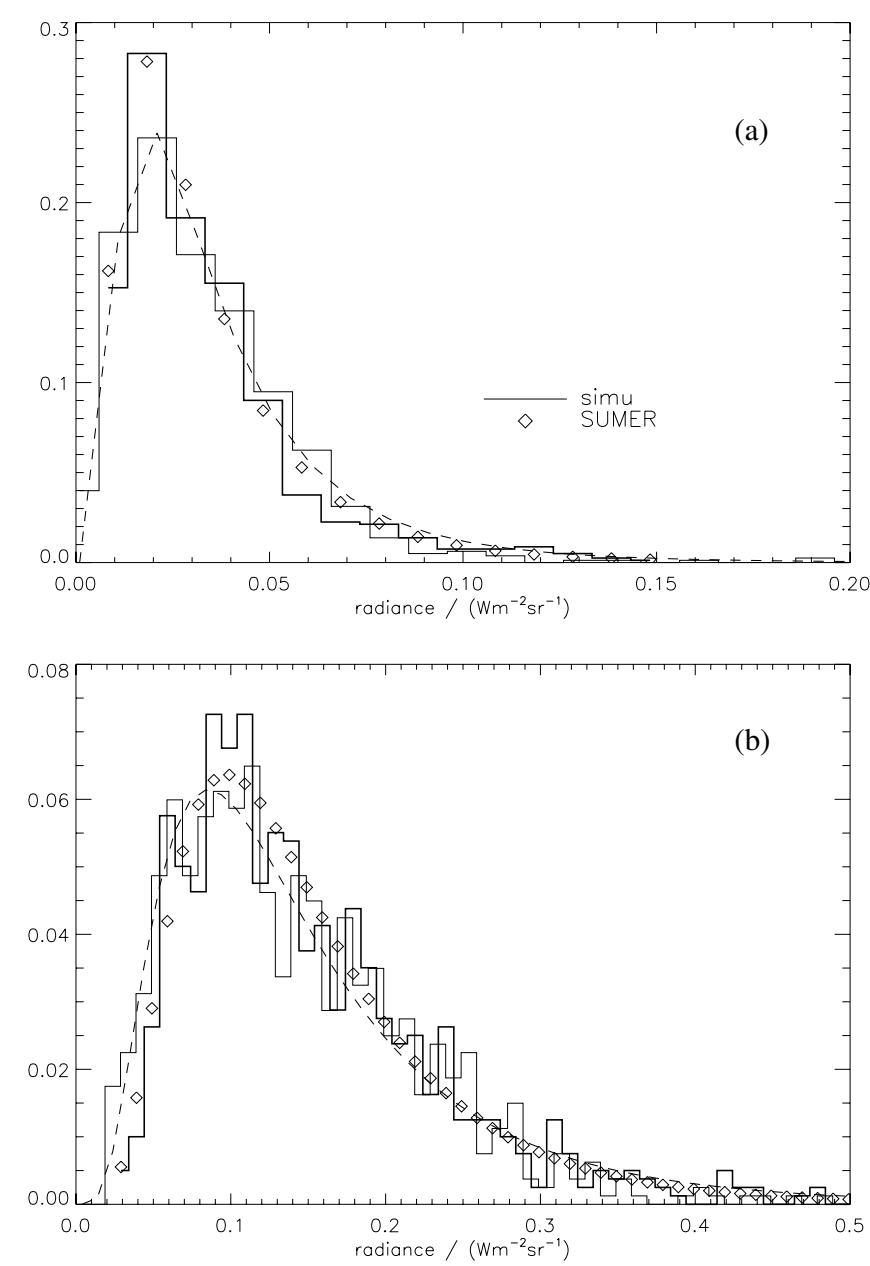

Fig. 11. Histograms and lognormal fits of the simulated (thin-line histogram and dashed curves, respectively) and SUMER (O Iv line at $79.0 \mathrm{~nm}$ ) measured (thick-line histogram and diamonds) radiances. a) from a time series in a very quiet area (cell interior), b) from a network sample of the same size $\left(1^{\prime \prime} \times 1^{\prime \prime}\right)$.

the single time series (see Figs. 12 and 13, and 16 and 17) and their histograms.

\subsection{Transition region}

Figure 11 shows two sample histograms from one-pixel time series (i.e., from a $1^{\prime \prime} \times 1^{\prime \prime}$ area on the solar surface) of the SUMER O IV data, the first from a darker area of the images, corresponding to the interior of a supergranule cell, the second from a brighter pixel (network). Also plotted in Fig. 11 are the histograms resulting from simulations with the parameters given in the top two rows of Table 3. 

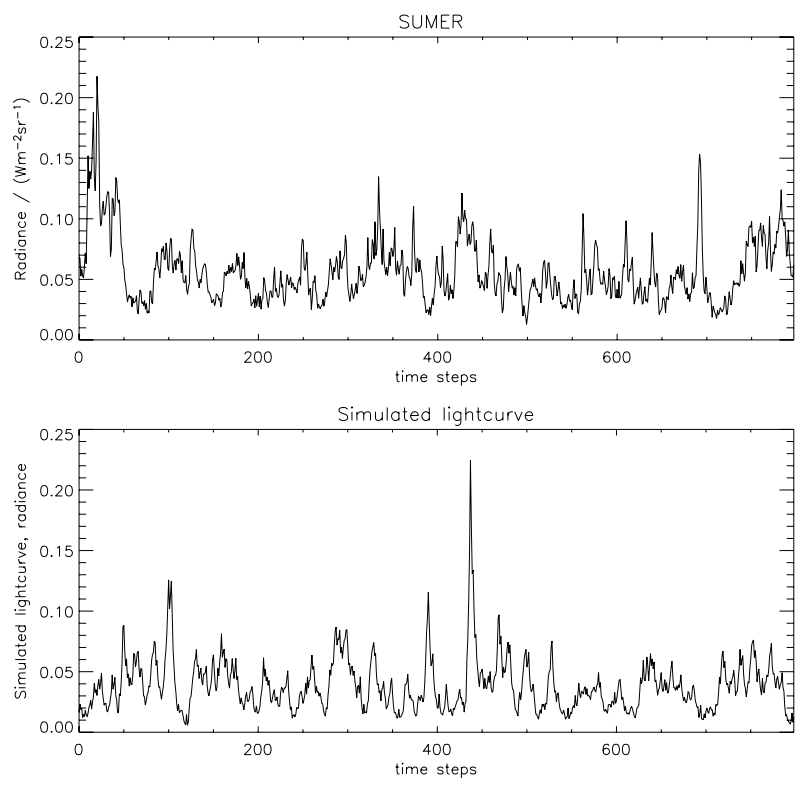

Fig. 12. SUMER time series of a darker ("cell") region (1 pixel) in the transition region O IV $79.0 \mathrm{~nm}$ line (upper frame), and a corresponding simulation (lower frame).
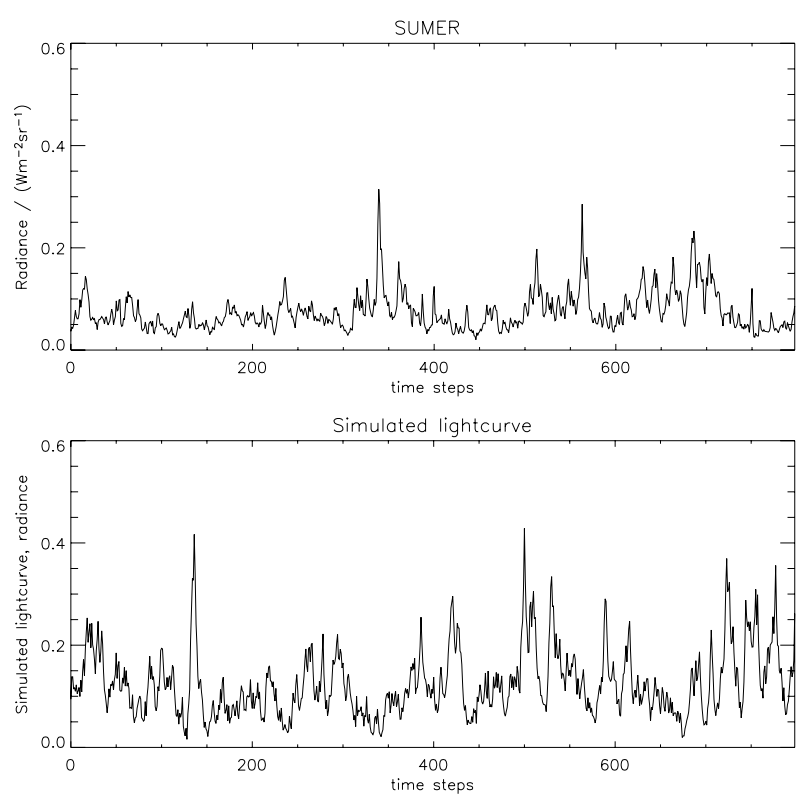

Fig. 13. SUMER time series of a brighter ("network") region (1 pixel) in the transition region $\mathrm{O}_{\text {IV }} 79.0 \mathrm{~nm}$ line, and a corresponding simulation.

The agreement between measured and modelled histograms is reasonable. The majority of the differences can be ascribed to the better statistics of the model.

Two examples of SUMER one-pixel time series of the O IV line are shown in Figs. 12 and 13, together with the corresponding simulations. The photon noise was on average $7 \%$ of the signal for the $\mathrm{O}$ IV data.

Figure 14 gives the measured and simulated power spectral densities, for both, the darker and the brighter region. The modelled and the observed power spectra now both display peaks at similar frequencies. It is noteworthy that the same model parameters reproduce both the network and the cell interior data. Only the amplitude of the input process had to be adjusted by setting the energy of the smallest flares. The large $\alpha$ value of 2.5 is obtained because the model attempts to reproduce all the
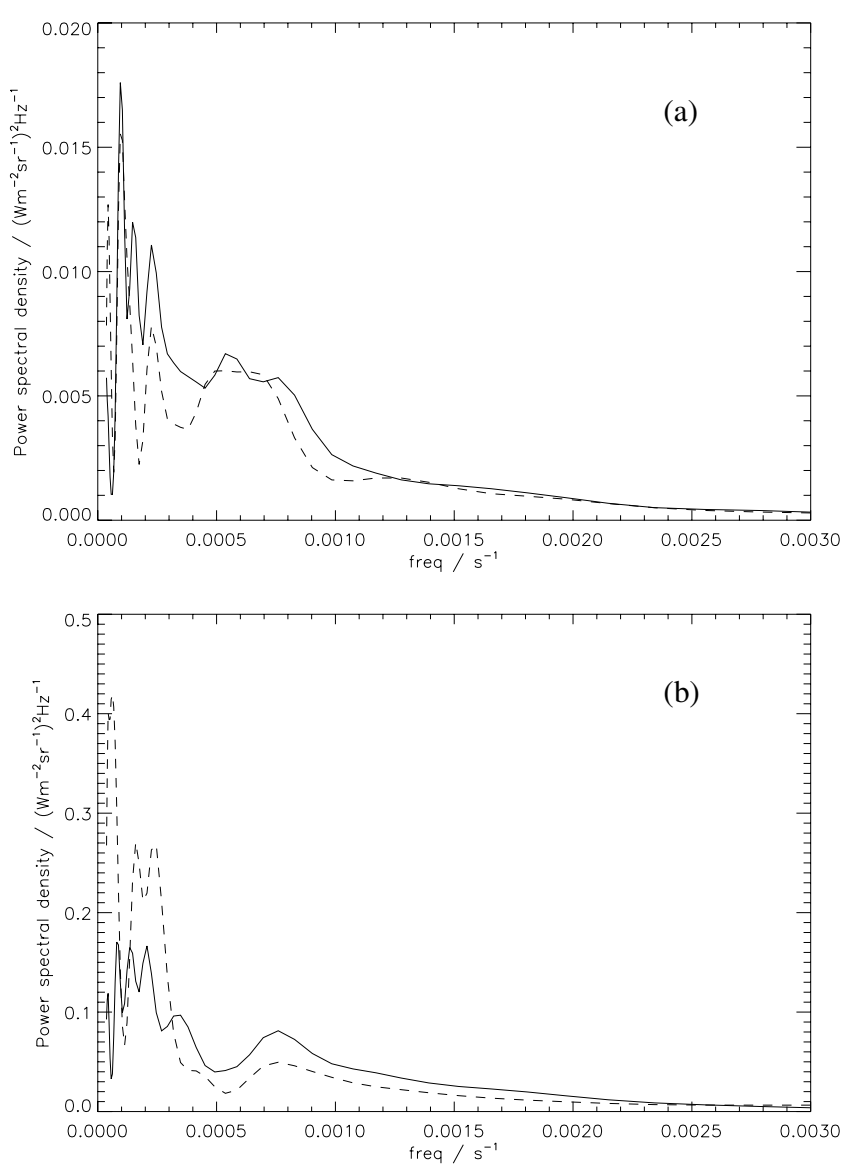

Fig. 14. Wavelet global power spectra for the time series shown in Fig. 12 (a), and Fig. 13 (b). The solid lines show the simulated spectrum, and the dashed lines give the spectrum of the SUMER time series.

diagnostics simultaneously and is not restricted to reproducing the brightenings but also the background.

\subsection{Corona}

The coronal time series and the corresponding histograms as well as power spectra recorded in cell interiors could be well represented by a weakly damped and low-frequent flare excitation, with the parameters given in the third row of Table 3. The correspondence between model and data can be judged from Figs. 15a, 16 and 18a. Roughly $9 \%$ photon noise was superposed on the modelled time series on average.

Satisfactory results for brighter regions could also be obtained, as illustrated in Figs. 15b, 17 and 18b. The parameters are listed in the last line of Table 3. $\tau_{\mathrm{d}}+\tau_{\mathrm{r}}$ needed to describe the Ne VIII time series and power spectra (Fig. 18) in the brighter parts of the quiet Sun are roughly 2.5 times larger than in the darker parts. The product $\left(\tau_{\mathrm{d}}+\tau_{\mathrm{r}}\right) / p_{\mathrm{f}}$, however, differs by a factor of only 1.5 . The difference in this product is sufficient to explain the relatively small difference in the mean intensity (Table 3), so that the same $y_{\min }$ is used for bright and dark regions.

\subsection{Physical parameters}

Clearly, our model is too simple to give, on its own, an accurate energy range of the flaring events that reproduce the data. 

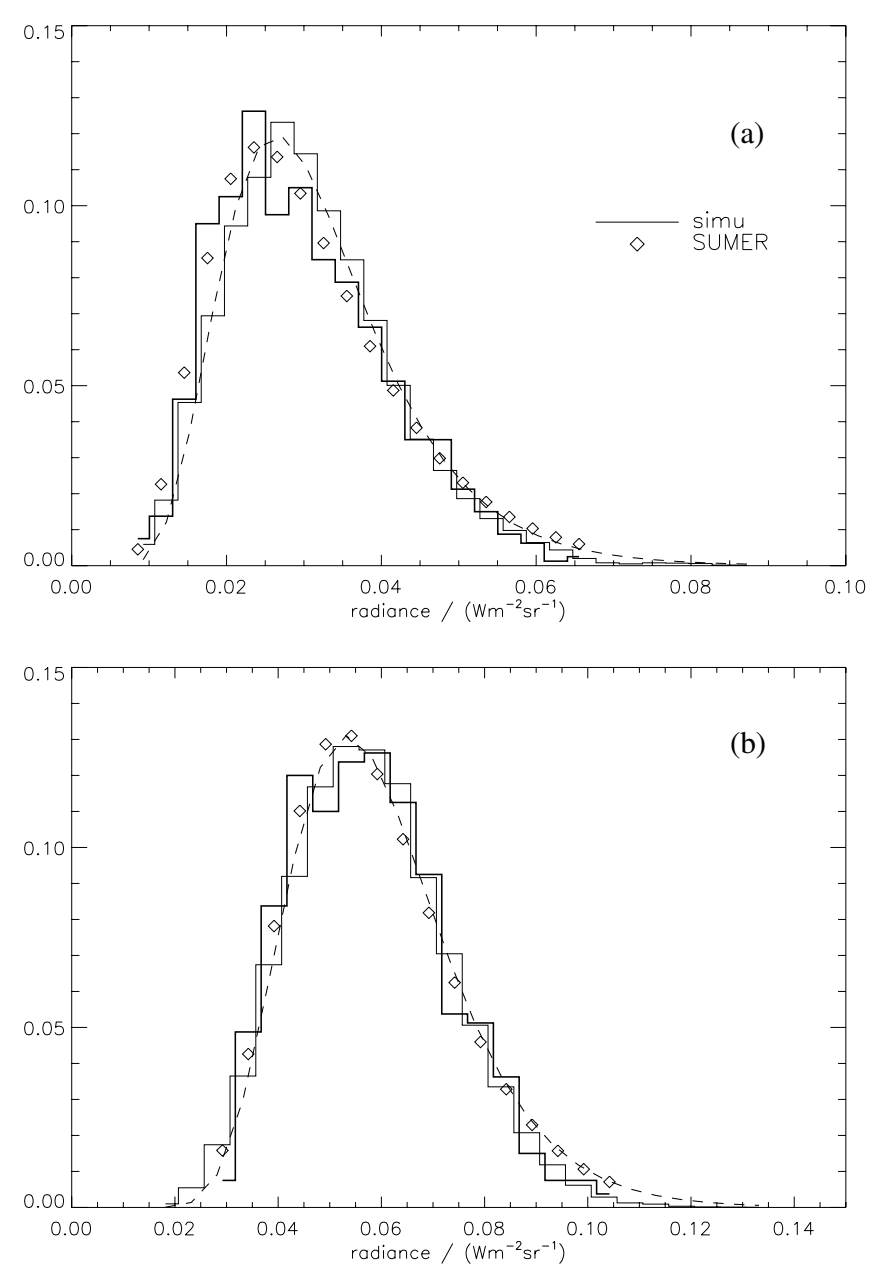

Fig. 15. Histograms and lognormal fits of the simulated (thin-line histogram and dashed curves, respectively) and SUMER (Ne VIII line at $77.0 \mathrm{~nm}$ ) measured (thick-line histogram and diamonds) radiances. a) from a time series in a dark pixel $\left(1^{\prime \prime} \times 1^{\prime \prime}\right)$, b) from a bright pixel.

However, with some restrictive assumptions a limited estimate can be made using Eq. (3).

The amplitudes listed in Table 3 correspond to flare energies between $6.4 \times 10^{16} \mathrm{erg}$ and $1.3 \times 10^{19} \mathrm{erg}$ for $\mathrm{O}$ IV and between $4.8 \times 10^{16} \mathrm{erg}$ and $1.6 \times 10^{17} \mathrm{erg}$ for Ne VIII for events of $1 \mathrm{~s}$ damping time covering an area of 1 square arcsecond (i.e., energy fluxes in the range between 12.6 and $2520 \mathrm{erg} \mathrm{s}^{-1} \mathrm{~cm}^{-2}$ for O IV and between 9.4 and $31.5 \mathrm{erg} \mathrm{s}^{-1} \mathrm{~cm}^{-2}$ for Ne VIII).

One grossly simplifying assumption that has already entered Eq. (3) concerns the geometry (radiation from a flat surface with no centre-to-limb variation). Another is that all events cover the same solar surface area, A. A third is that all events have the same temperature (or rather the same temperature-density relationship), so that a single $q$ value can be employed for all. We do not attempt to compute $q$, so that we only estimate the energy radiated in the observed spectral line. For the area $A$ we assume a size of $10^{13} \mathrm{~m}^{2}$, which would roughly be 20 SUMER pixels (Aschwanden et al. (2000) found sizes of $4-200 \times 10^{12} \mathrm{~m}^{2}$ using TRACE data), and also adopt durations of 200 to $2000 \mathrm{~s}$. Under these assumptions we obtain a total range of energies between $2.52 \times 10^{20}$ erg to $5 \times 10^{24}$ erg for individual events in the $\mathrm{O}$ IV line and $1.88 \times 10^{20} \mathrm{erg}$ to $6.3 \times 10^{21} \mathrm{erg}$ for events in the $\mathrm{Ne}$ VIII line. Of course, this energy radiated away by only single
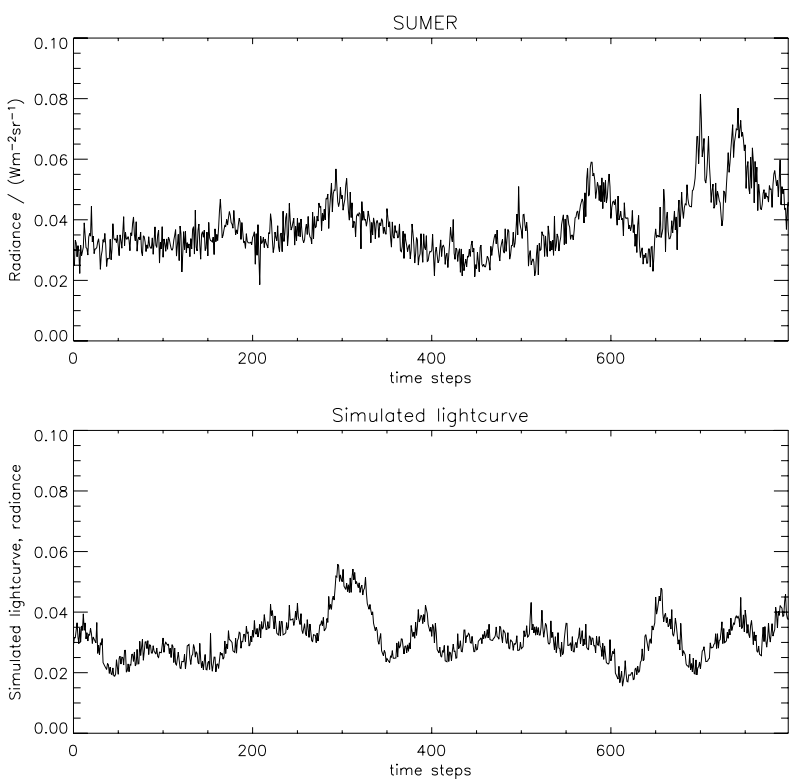

Fig. 16. SUMER time series of a darker region (1 pixel) in the upper transition region/coronal line $77.0 \mathrm{~nm}$ (Ne VIII), and a corresponding simulation.
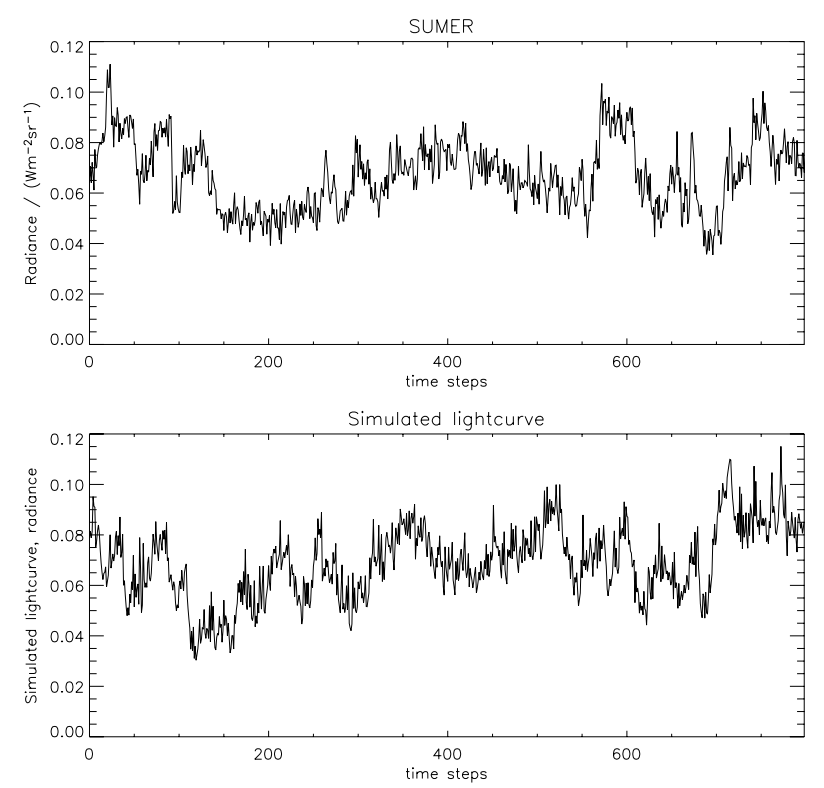

Fig. 17. SUMER time series of a brighter region (1 pixel) in the upper transition region/coronal line $77.0 \mathrm{~nm}$ (Ne vIII), and a corresponding simulation.

spectral lines is still too low by several orders of magnitude to make up for the upper chromospheric or coronal energy losses given, e.g., by Withbroe \& Noyes (1977).

From the flaring frequencies given in Table 3, again assuming an event size of 20 SUMER pixels, one can obtain a rough estimate of the number of such events on the total solar surface. To reproduce the SUMER time series in the transition region line and bright corona including the background, it would need roughly 7600 events per second. 2000 events per second can generate the radiation in the darker areas of the Ne VIII line. Given all the uncertainties entering the above estimates we prefer to restrict ourselves to order of magnitude estimates: our model suggests a nano-flaring rate of $10^{3}$ to $10^{4}$ events per second. These values can be compared with the number of blinkers estimated 

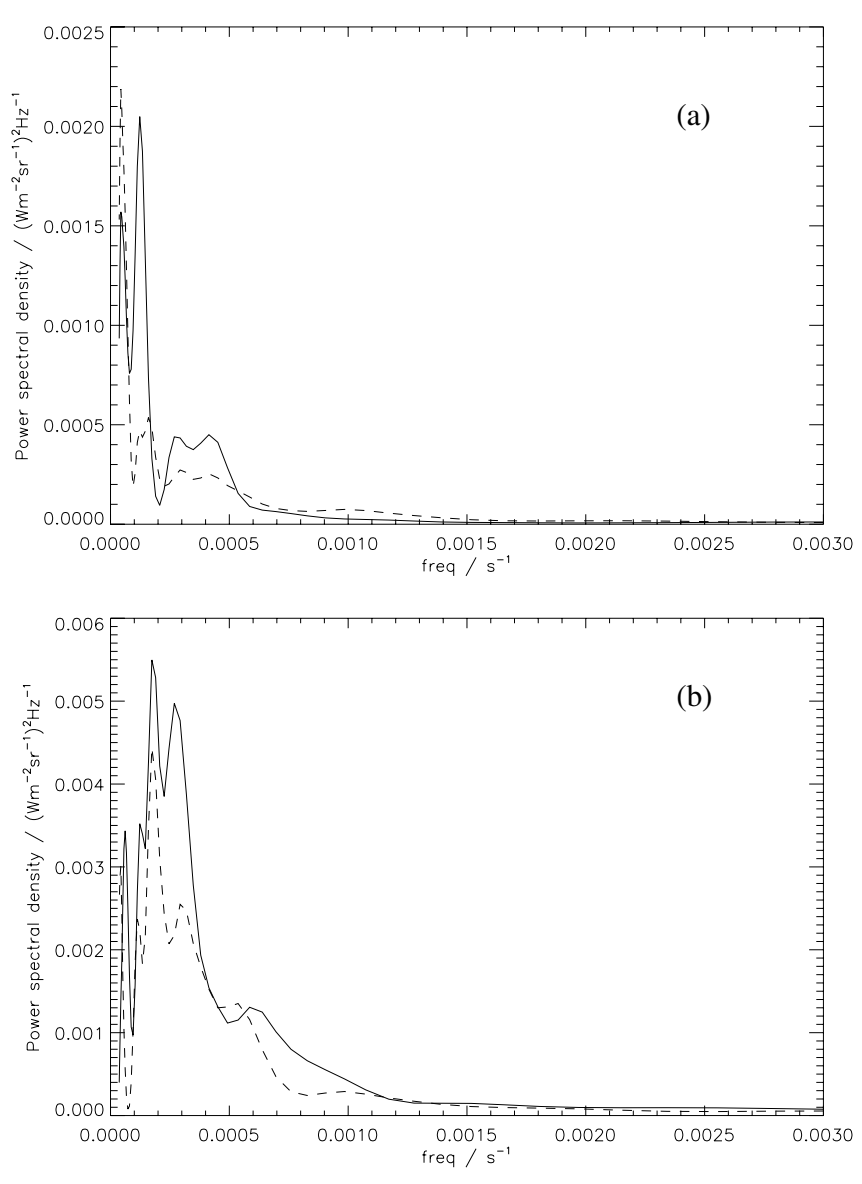

Fig. 18. Wavelet global power spectra for the time series shown in Fig. 16 (a), and Fig. 17 (b). The solid lines show the simulated spectrum, and the dashed lines give the spectrum of the SUMER time series.

for the total solar surface (50 events/s, Brković et al. 2000) and that of explosive events (500 events/s, Innes et al. 1997).

To estimate the total available flare input for heating one would need to know or extrapolate these values to the entire spectrum, assuming as precise areas as possible (geometry, filling factor) and prescribe which fraction of the total released energy is radiated away (and not, e.g., employed to accelerate the solar wind). Here we take a simpler approach. In order to get an idea of the energy released by a typical brightening, we use the total coronal radiative energy loss of the quiet Sun $F_{r}=10^{5} \mathrm{erg} \mathrm{cm}^{-2} \mathrm{~s}^{-1}$ (corresponding to $6.07861 \times 10^{27} \mathrm{erg} \mathrm{s}^{-1}$ over the entire solar surface area) given by Withbroe \& Noyes (1977). Assuming 7600 events per second, we get a mean radiative loss of $8 \times 10^{23}$ erg per event which is an integral over the full spectrum and an average over the darker and brighter network areas.

If we further assume the same power-law exponent $\alpha=2.5$ over the entire spectrum, we can estimate the lower energy limits of the flaring events obtained from our analysis from Eq. (13),

$m_{\text {all }}=3 \frac{\left(y_{\max }^{-0.5}-y_{\min }^{-0.5}\right)}{\left(y_{\max }^{-1.5}-y_{\min }^{-1.5}\right)} \longrightarrow y_{\min } \approx 8 \times 10^{23} \mathrm{erg}$

for 7600 events per second. Here we have further assumed that $y_{\max } \gg y_{\min }$. For 2000 events per second (as was estimated for the darker parts of the corona with smaller flaring frequency and much larger damping time), the average flare energy would approximate $3.04 \times 10^{24} \mathrm{erg}$. These values would make the brightening events in our analysis nano- and microflares, with the smallest ones being rather small nanoflares or large picoflares, depending on where the lower energy limit for flares is set. Parker (1988) estimated values of around $1.6 \times 10^{24} \mathrm{erg}$ per nanoflare event.

\section{Discussion and conclusions}

In this paper we consider the diagnostic content of radiance time series and simulate them with the help of a simple stochastic model. The radiances of time series measured by SUMER in the quiet Sun are distributed following a lognormal function for every spatial point. The properties of these distributions reflect those found by Pauluhn et al. (2000) in the sense that transition region radiation shows broader, more asymmetric distributions, while the coronal radiance exhibits a narrower, more symmetric distribution. These results show that quiet-Sun radiances exhibit a lognormal distribution irrespective of whether we sample spatially (Pauluhn et al. 2000) or temporally (this paper), although in the former case we are mixing radiation from network and supergranule cells, while in the latter case we are considering these features separately.

The shapes of the lognormals (Figs. 11 and 15) emphasize the lack of points around a radiance of zero, with lines at higher temperatures showing this effect more strongly. In the past such a behaviour has often been interpreted to indicate the superposition of a more or less steady background and overlying peaks produced by discrete energy releases, e.g., by microflares. Here we assume that all the radiance in the observed lines is caused by micro- and nanoflares, so that any background is due to a superposition of many small brightenings.

We have developed a model which attempts to explain the properties of the observed time series assuming that all the radiation coming from the transition region and the corona is due to the cooling of gas that had been heated by nanoflares. From the multiplicative version of the central limit theorem it follows that the product of many independent, identically distributed, positive random variables has approximately a lognormal distribution. (For a discussion of the special features of the lognormal distribution function see also, e.g., Limpert et al. 2001.) For a lognormal distribution to form in a simulated time series, the input process has to be positive definite (thus, a Gaussian with zero mean as input distribution does not produce a lognormal). By the positive input process, the asymmetric shape is ensured.

The shape and scale of the resulting lognormal are strongly dependent on the ratio of the damping to the excitation process. The higher the flaring frequency and the longer the damping time, the more symmetric the resulting lognormal becomes (i.e., the $\sigma$ values which determine the shape of the lognormal become smaller and the distribution becomes more Gaussian-like) and vice versa. The scaling of the distribution, which is its extension in (peak-) height and width and is given by the parameter $\mu$ of the lognormal, increases with damping time (i.e., with decreasing damping) and with flaring frequency. Consequently, if many small brightenings overlap, they produce a stronger "background" emission than fewer brightenings lying further apart. It has to be noted that the SUMER time series could be reproduced in a model employing the same damping time for weak and strong flares.

An important part of this work has been to identify representations of the data which can serve as diagnostics to constrain a microflare model and to determine the free parameters, in particular the power-law exponent $\alpha$ of the flare amplitudes or 
energies. The combination of the power spectrum of the radiance time series and the distribution function, together with the time series itself are found to constrain the free parameters of the model, including $\alpha$, relatively well. The most important result of this paper is that simulations that best reproduce the data had power-law exponents $\alpha$ of around 2.5. We stress that we have not carried out a thorough uniqueness analysis here, although numerous attempts to reproduce the data with $\alpha<2$ were unsuccessful. Note also that this value is obtained assuming that all (nano-)flares have the same geometry and cover the same area. The large $\alpha$ is determined by the need to reproduce the background emission seen in the transition region and the corona. Smaller $\alpha$ values give too much weight to single larger flares which do not reproduce the purely quiet time series (cf., Fig. 9). By assuming that all micro- and nanoflares cover the same surface area of $10^{13} \mathrm{~m}^{2}$ following Aschwanden et al. (2000), we find a nanoflaring rate of $10^{3}-10^{4} \mathrm{~s}^{-1}$.

An interesting result of the current investigations is that the damping time scales of the simulations reproducing the coronal time series are found to be significantly larger than in the transition region (by a factor of 4 to 8 ). A simple estimate of the radiative cooling times based on the Rosner formula gives (cf., Rosner et al. 1978):

$\tau_{\mathrm{rad}}=3 \frac{1.38 \times 10^{-16}}{n_{\mathrm{e}} \xi T^{a-1}}$.

For the temperature of formation of the O IV line, $T=1.5 \times$ $10^{5} \mathrm{~K}, \xi=6.3 \times 10^{-22}, a=0$, while for $T=6 \times 10^{5} \mathrm{~K}$, appropriate for Ne VIII, $\xi=3.98 \times 10^{-11}, a=-2$. If we assume a constant pressure of $0.3 \mathrm{dyn} \mathrm{\textrm {cm } ^ { - 2 }}\left(=0.03 \mathrm{~N} \mathrm{~m}^{-2}\right)$, we obtain a ratio of $\tau_{\text {rad }}(\mathrm{Ne}$ VIII $) / \tau_{\text {rad }}\left(\mathrm{O}_{\text {IV }}\right) \approx 100$, which is in the same direction, as that obtained from the analysis, although an order of magnitude larger.

The similar flaring probability, $p_{\mathrm{f}}$, obtained for (the brighter part of the quiet) corona and transition region does not allow us to determine whether the brightnings in the corona and the transition region are due to the same or to different flaring events based on this analysis.

Further work is needed, both to demonstrate the uniqueness of the deduced parameters, in particular $\alpha$, to increase the amount of realism in the model, and to consider more data. The first may be achieved by automating the process of finding a best statistical fit to the data (i.e., best simultaneous fit to the distribution function and the power spectrum), possibly coupled to a Monte Carlo simulation or involving genetic algorithms. An analysis of lines sampling higher temperatures is required in order to reveal the behaviour of hot coronal gas. There is also a need to statistically simulate time series of images. Such data may also provide some information on the size distribution of flares. Since a single time series belonging to a particular pixel may sample only some spatially limited parts of particular brightenings, the parameters obtained by statistically reproducing it with a 1-d model need not be entirely representative of the real Sun. Thus, higher-dimensional models could be of great advantage.

Finally, an application of the analysis developed here to active regions could reveal similarities and differences between the flaring processes in active regions and the quiet Sun.

Acknowledgements. SOHO is a project of international cooperation between ESA and NASA. The SUMER project is financially supported by Deutsches Zentrum für Luft- und Raumfahrt (DLR), the Centre National d'Etudes Spatiales (CNES), the National Aeronautics and Space Administration (NASA), and the ESA PRODEX programme (Swiss contribution). Wavelet software was provided by C. Torrence and G. Compo, and is available at URL: http://paos.colorado.edu/research/wavelets/. We are grateful to Davina Innes, Luca Teriaca and an anonymous referee who made valuable suggestions and helped substantially improve the manuscript.

\section{References}

Arzner, K., \& Güdel, M. 2004, ApJ, 602, 363

Aschwanden, M. J., Tarbell, T. D., Nightingale, R. W., et al. 2000, ApJ, 535, 1047

Aschwanden, M. J., \& Parnell, C. E. 2002, ApJ, 572, 1048

Audard, M., Güdel, M., Drake, J. J., \& Kashyap, V. L. 2000, ApJ, 541, 396

Brković, A., Solanki, S. K., \& Rüedi, I. 2001, A\&A, 373, 1056

Charbonneau, P. 2002, NCAR Technical Note TN-450+IA

Charbonneau, P., \& Knapp, B. 1998, NCAR Technical Note TN-418+IA

Datlowe, D. W., Elcan, M. J., \& Hudson, H. S. 1974, Sol. Phys., 39, 155

Güdel, M., Audard, M., Drake, J. J., Kashyap, V. L., \& Guinan, E. F. 2003, ApJ, 582,423

Harrison, R. A. 1997, Sol. Phys., 175, 467

Honerkamp, J. 1990, Stochastische Dynamische Systeme (Weinheim, Germany: $\mathrm{VCH})$

Innes, D. E., Inhester, B., Axford, W. I., \& Wilhelm, K. 1997, Nature, 386, 811

Krucker, S., \& Benz, A. O. 1998, ApJ, 501, L213

Limpert, E., Stahel, W. A., \& Abbt, M. 2001, BioScience, 51/5, 341

Lin, R. P., Schwartz, R. A., Kane, S. R., Pelling, R. M., \& Hurley, K. C. 1984, ApJ, 283, 421

Lu, E. T., \& Hamilton, R. J. 1991, ApJ, 380, L89

Mitra-Kraev, U., \& Benz, A. O. 2000, A\&A, 373, 318

Parker, E. N. 1988, ApJ, 330, 474

Parnell, C. E., \& Jupp, P. E. 2000, ApJ, 529, 554

Pauluhn, A., Solanki, S. K., Rüedi, I., Landi, E., \& Schühle, U. 2000, A\&A, 362, 737

Phillips, K. J. H., \& Dwivedi, B. N. 2003, Dynamic Sun (Cambridge University Press), 335

Rosner, R., Tucker, W. H., \& Vaiana, G. S. 1978, ApJ, 220, 643

Solanki, S. K. 2002, ISSI Sci. Rep. SR-002, ed. A. Pauluhn, M. C. E. Huber, \& R. von Steiger, 1

Tandberg-Hanssen, E., \& Emslie, A. G. 1988, The Physics of Solar Flares (Cambridge, UK: Cambridge University Press)

Torrence, C., \& Compo, G. P. 1998, Bull. Amer. Meteor. Soc., 79, 61

Vekstein, G. E., \& Jain, R. 2003, Plasma Phys. Control. Fusion, 45, 535

Winebarger, A. R., Emslie, A. G., Mariska, J. T., \& Warren, H. P. 2002, ApJ, 565,1298

Wilhelm, K., \& Kalkofen, W. 2003, A\&A, 408, 1137

Wilhelm, K., Curdt, W., Marsch, E., et al. 1995, Sol. Phys., 162, 189

Withbroe, G. L., \& Noyes, R. W. 1977, ARA\&A, 15, 363 